\title{
Measurement of Single Top Production at the Tevatron
}

\author{
Dominic Hirschbühl \\ on behalf of the CDF and DØ Collaborations \\ Institut für Experimentelle Kernphysik \\ Universität Karlsruhe, Wolfgang-Gaede-Straße 1, 76131 Karlsruhe
}

\begin{abstract}
This article reports on first evidence for single top-quark production by the CDF and $\mathrm{D} \varnothing$ collaboration at the Tevatron. Both experiments have measured the cross section with more the 3 standard deviations significance using a data set that corresponds to an integrated luminosity of $2.2 \mathrm{fb}^{-1}$ and $0.9 \mathrm{fb}^{-1}$, respectively. Also direct measurements of the CKM matrix element $\left|V_{t b}\right|$, that describes the $W t b$ coupling, are performed.
\end{abstract}

\section{Introduction}

In $p \bar{p}$ collisions at the Tevatron top quarks are mainly produced in pairs via the strong force. However, the standard model also predicts the production of single top-quarks by the weak interaction via the $s$ - or $t$-channel exchange of an off-shell $W$ boson.

The single top-quark production cross-section is predicted to $\sigma_{\mathrm{s}+\mathrm{t}}=2.9 \pm 0.4 \mathrm{pb}$ for a top mass of $175 \mathrm{GeV} / c^{2}[2]$ which is about $40 \%$ of the top-antitop pair production cross section. The main obstacle in finding single-top is however not the production rate of the signal but the large background rate. After all selection requirements are imposed, the signal to background ratio is approximately $1 / 16$. This challenging, background-dominated dataset is the main motivation for using multivariate techniques.

\section{Event selection and background estimation}

The analyses performed by CDF are based on the same event selection and use the same Run II data set corresponding to an integrated luminosity of $2.2 \mathrm{fb}^{-1}$. The analyses performed by $\mathrm{D} \emptyset$ are also based on a common event selection using data corresponding to an integrated luminosity of $0.9 \mathrm{fb}^{-1}$. The event selection exploits the kinematic features of the signal final state, which contains a top quark, a bottom quark, and possibly additional light quark jets. To reduce multijet backgrounds, the $W$ boson originating from the top quark is required to have decayed leptonically. One therefore demands a single, isolated, high-energy electron or muon and large missing transverse energy from the undetected neutrino. The remaining backgrounds belong to the following categories: $W b \bar{b}, W c \bar{c}, W c$, mistags, where light quarks are misidentified as heavy flavor jets, QCD-multijet production, where a jet is misreconstructed as an electron or a heavy-flavor quark decays to a lepton that is misidentified as isolated from the jet, and $W W, W Z, Z Z$ production. The CDF analyses select only events with two or three jets, whereas $D \varnothing$ includes also four-jet events. We remove a large fraction of the backgrounds by demanding that at least one of these jets should be tagged as a $b$-quark jet. The number of expected and observed events are listed in Table 1.

\subsection{Cross Section Measurement at D}

$\mathrm{D} \varnothing$ uses three different multivariate techniques to extract the signal from data: Boosted Decision Trees (BT), Matrix Element discriminants (ME) and Bayesian Neural Networks 


\begin{tabular}{|lcc||ccc|}
\hline \multicolumn{2}{|c|}{ CDF $2.2 \mathrm{fb}^{-1}$} & \multicolumn{3}{c|}{ DØ $0.9 \mathrm{fb}^{-1}$} \\
Source & 2 jets & 3 jets & 2 jets & 3 jets & 4 jets \\
\hline s-channel & $41.2 \pm 5.9$ & $13.5 \pm 1.9$ & $16 \pm 3$ & $8 \pm 2$ & $2 \pm 1$ \\
t-channel & $62.1 \pm 9.1$ & $18.3 \pm 2.7$ & $20 \pm 4$ & $12 \pm 3$ & $4 \pm 1$ \\
\hline$W+b b$ & $461.6 \pm 139.7$ & $141.1 \pm 42.6$ & $261 \pm 55$ & $120 \pm 24$ & $35 \pm 7$ \\
$W+c \bar{c}, W+c$ & $395.0 \pm 121.8$ & $108.8 \pm 33.5$ & $151 \pm 31$ & $85 \pm 17$ & $23 \pm 5$ \\
$W+j j$ & $339.8 \pm 56.1$ & $101.8 \pm 16.9$ & $119 \pm 25$ & $43 \pm 9$ & $12 \pm 2$ \\
QCD-multijet & $59.5 \pm 23.8$ & $21.3 \pm 8.5$ & $95 \pm 19$ & $77 \pm 15$ & $29 \pm 6$ \\
Diboson & $63.2 \pm 6.3$ & $21.5 \pm 2.2$ & & & \\
$Z+$ jets & $26.7 \pm 3.9$ & $11.0 \pm 1.6$ & & & \\
\hline Total background & $1491.8 \pm 268.6$ & $754.8 \pm 91.3$ & $686 \pm 41$ & $460 \pm 39$ & $253 \pm 38$ \\
Observed & 1535 & 712 & 697 & 455 & 246 \\
\hline
\end{tabular}

Table 1: Expected number of signal and background events and total number of events observed in $2.2 \mathrm{fb}^{-1}$ in the CDF single-top dataset (left) and in $0.9 \mathrm{fb}^{-1}$ in the DØ singletop dataset (right)

(BNN). In Figure 1 the three output distributions of simulated events, scaled to the expected number of events, compared with the measured values are shown. To measure the single
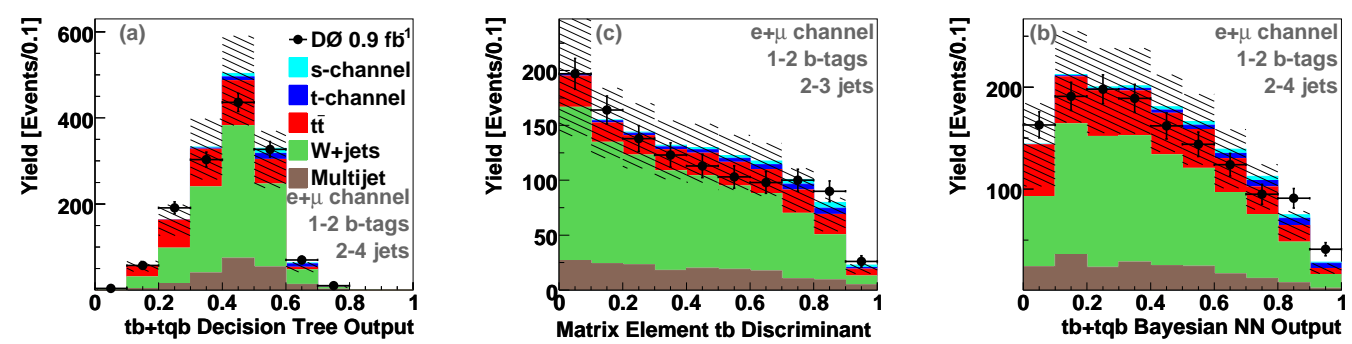

Figure 1: Output distribution of the Boosted Decision Trees analysis (left), of the $t b$ Matrix Element discriminant (middle) and of the Bayesian Neural Networks (right).

top-quark production cross section the full output distributions are fed into a Bayesian statistical analysis. The expected and observed cross section results are summarized in Figure 2. The given uncertainties include statistical as well as systematic uncertainties. In order to measure the significance, ensemble tests including only background sources with all the uncertainties on the background model are utilized. A combination of the three analyses is performed using the best linear unbiased estimator (BLUE) [3] which yield a combined cross section of $\sigma=4.8 \pm 1.3 \mathrm{pb}$ corresponding to a significance of $0.0027 \%$ or 3.5 standard deviations. The result of the separate search is given in Figure 2. These results have recently been published [4].

\subsection{Cross Section Measurement at CDF}

CDF performs also three different multivariate analyses: Likelihood discriminants (LF), Matrix Element discriminants (ME) and Neural Networks (NN). For a combination the 


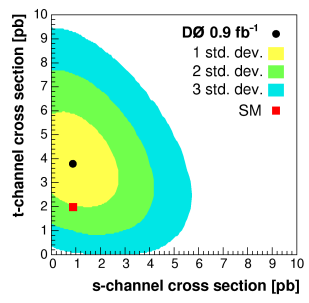

\begin{tabular}{cccc}
\hline & Cross section $[\mathrm{pb}]$ & Exp. & Obs. \\
\hline DT & $4.9 \pm 1.4$ & $2.1 \sigma$ & $3.4 \sigma$ \\
ME & $4.8_{-1.4}^{1.6}$ & $1.9 \sigma$ & $3.2 \sigma$ \\
BNN & $4.4_{-1.4}^{1.6}$ & $2.2 \sigma$ & $3.1 \sigma$ \\
\hline \hline BLUE & $4.7 \pm 1.3$ & $2.3 \sigma$ & $3.6 \sigma$ \\
\hline
\end{tabular}

Figure 2: Posterior probability density for the BDT analysis as a function of the $s$-channel and $t$-channel cross section. The table gives the measured single top-quark production crosssections with the corresponding expected and observed significance.

output of the different analyses is fed to an evolved Neural Network (NEAT) [5] producing a super discriminant. Additionally, the single-cross section measurement are combined using BLUE. One of the variables used in all CDF single top-quark analyses is the output of a neural network flavor separator [6]. In Figure 3 the distribution of this variable is shown for the observed events in $2.2 \mathrm{fb}^{-1}$. The flavor separator gives an additional handle to reduce the

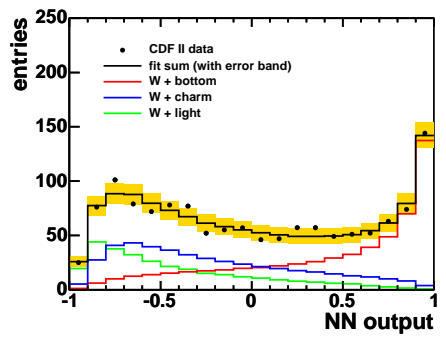

\begin{tabular}{cccc}
\hline & Cross section $[\mathrm{pb}]$ & Exp. & Obs. \\
\hline $\mathrm{LF}$ & $1.8_{-0.8}^{+0.9}$ & $3.4 \sigma$ & $2.0 \sigma$ \\
$\mathrm{ME}$ & $2.2_{-0.7}^{+0.8}$ & $4.5 \sigma$ & $3.4 \sigma$ \\
$\mathrm{NN}$ & $2.0_{-0.8}^{+0.9}$ & $4.4 \sigma$ & $3.2 \sigma$ \\
\hline \hline NEAT & $2.1_{-0.6}^{+0.7}$ & $5.1 \sigma$ & $3.7 \sigma$ \\
BLUE & $2.1 \pm 0.7$ & $4.7 \sigma$ & $3.7 \sigma$ \\
\hline \hline
\end{tabular}

Figure 3: Output distribution of the flavor separator. The table gives the measured single top-quark production cross-sections with the corresponding expected and observed significance.

large background components where no real $b$ quarks are contained, which amount to about $50 \%$ in the observed $W$ events with 2 jets, even after imposing the requirement that one jet is identified by the secondary vertex tagger of CDF [7]. The signal significance is calculate using the probability of the background-only discriminant to fluctuate to the observed data or more.Hereby all sources of systematic uncertainties are included. The results including the most probable value of the combined cross section are shown in Figure 3.

\section{Measurement of the CKM Matrix element $\left|V_{t b}\right|$}

A limit of the CKM matrix element $\left|V_{t b}\right|$ can be determined using the posterior probability function under the assumption that $\left|V_{t d}\right|^{2}+\left|V_{t s}\right|^{2} \ll\left|V_{t b}\right|^{2}$ and assuming a flat prior of $\left|V_{t b}\right|^{2}$ between 0 and 1. D $\varnothing$ set an lower limit of $0.68 @ 95 \%$ C.L. based on the measurement of the BDT analysis. CDF uses the result of the NEAT network and sets an upper limit of $0.66 @ 95 \%$ C.L. 

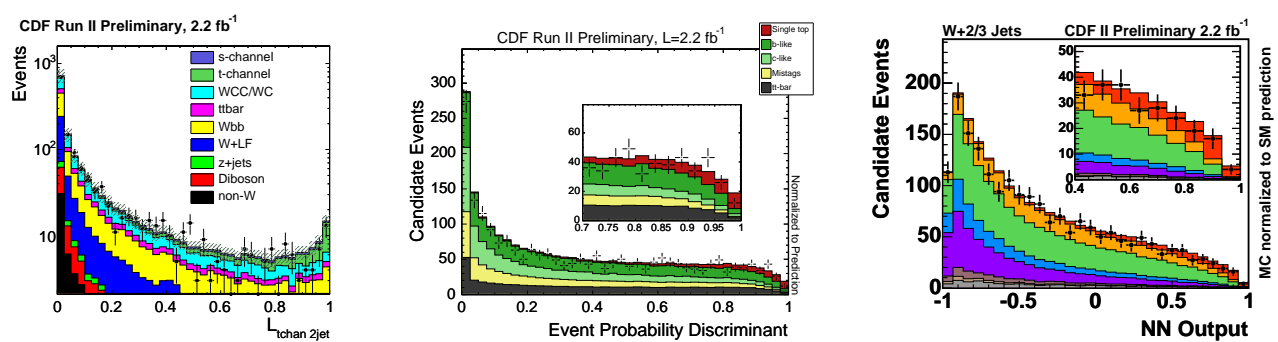

Figure 4: Output distribution of the Likelihood Function analysis (left), of the Matrix Element discriminant (middle) and of the Neural Network analysis (right).
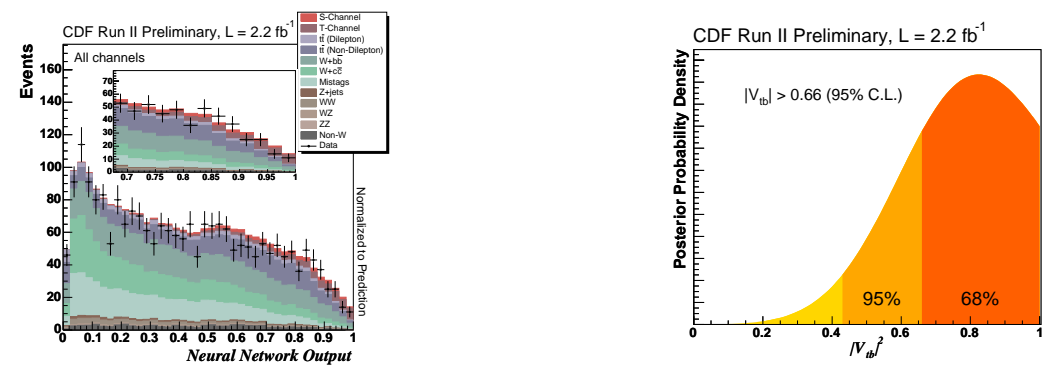

Figure 5: Output distribution of the combined NEAT network (left) and the posterior probability density function.

\section{Conclusions}

Both collaborations has performed searches for standard model single top-quark production using a data set corresponding to an integrated luminosity of $0.9 \mathrm{fb}^{-1}(\mathrm{D} \emptyset)$ and $2.2 \mathrm{fb}^{-1}(\mathrm{CDF})$ and have found evidence with more than $3 \sigma$ significance. Also a direct measurement of the CKM matrix element $\left|V_{t b}\right|$ has been performed. As the Tevatron collects more data, both experiments continue to improve their analyses.

\section{References}

[1] Slides: http: //indico. cern. ch/contributionDisplay. py? contribId=111\&sessionId=15\&conf Id $=24657$

[2] B. W. Harris et al., Phys. Rev. D66, 054024 (2002); Z. Sullivan, Phys. Rev. D70, 114012 (2004); J. Campbell, R.K. Ellis, F. Tramontano, Phys. Rev. D70, 094012 (2004); N. Kidonakis, Phys. Rev., D74, 114012 (2006).

[3] L. Lyons, D. Gibaut and P. Clifford, Nucl. Instrum. Meth. A 270, 110 (1988).

[4] V. M. Abazov et al. [D0 Collaboration], Phys. Rev. D 78, 012005 (2008)

[5] K. O. Stanley and R. Miikkulainen, Evolutionary Computation 10 (2) 99-127 (2002).

[6] S. Richter, "Search for electroweak single top-quark production with the CDF II experiment," FERMILAB-THESIS-2007-35

[7] D. Acosta et al. (CDF Collaboration), Phys. Rev. D71, 052003. 